\title{
Multicentre chest computed tomography standardisation in children and adolescents with cystic fibrosis: the way forward
}

\author{
Wieying Kuo ${ }^{1,2}$, Mariette P.C. Kemner-van de Corput ${ }^{2}$, Adria Perez-Rovira ${ }^{1,3}$, \\ Marleen de Bruijne ${ }^{3,4}$, Isabelle Fajac ${ }^{5,6}$, Harm A.W.M. Tiddens ${ }^{1,2}$ and \\ Marcel van Straten $^{2}$ on behalf of the ECFS-CTN/SCIFI CF study group ${ }^{7}$
}

\begin{abstract}
Affiliations: 'Dept of Pediatric Pulmonology, Erasmus MC - Sophia Children's Hospital, Rotterdam, The Netherlands. ${ }^{2}$ Dept of Radiology, Erasmus MC, Rotterdam, The Netherlands. ${ }^{3}$ Biomedical Imaging Group Rotterdam, Depts of Radiology and Medical Informatics, Erasmus MC, Rotterdam, The Netherlands. ${ }^{4}$ Dept of Computer Science, University of Copenhagen, Copenhagen, Denmark. ${ }^{5}$ Université Sorbonne Paris Cité, Faculté de Médecine Paris Descartes, Paris, France. ${ }^{6}$ AP-HP, Hôpitaux Universitaires Paris Centre, Hôpital Cochin, Service d'Explorations Fonctionnelles, Paris, France. ${ }^{7} \mathrm{~A}$ list of the SCIFI CF members can be found in the Acknowledgements section.
\end{abstract}

Correspondence: Marcel van Straten, Erasmus MC, s-Gravendijkwal 230, 3015 CE Rotterdam, The Netherlands. E-mail: marcel.vanstratenderasmusmc.nl

ABSTRACT Progressive cystic fibrosis (CF) lung disease is the main cause of mortality in CF patients. CF lung disease starts in early childhood. With current standards of care, respiratory function remains largely normal in children and more sensitive outcome measures are needed to monitor early CF lung disease. Chest CT is currently the most sensitive imaging modality to monitor pulmonary structural changes in children and adolescents with CF. To quantify structural lung disease reliably among multiple centres, standardisation of chest CT protocols is needed. SCIFI CF (Standardised Chest Imaging Framework for Interventions and Personalised Medicine in CF) was founded to characterise chest CT image quality and radiation doses among 16 participating European CF centres in 10 different countries. We aimed to optimise CT protocols in children and adolescents among several CF centres. A large variety was found in CT protocols, image quality and radiation dose usage among the centres. However, the performance of all CT scanners was found to be very similar, when taking spatial resolution and radiation dose into account. We conclude that multicentre standardisation of chest CT in children and adolescents with CF can be achieved for future clinical trials.

@ERSpublications

Multicentre chest CT standardisation is necessary and feasible to maximise image quality and reduce radiationhttp://ow.ly/ZfsaE

This article has supplementary material available from erj.ersjournals.com

Received: Sept 252015 | Accepted after revision: March 022016 | First published online: April 132016

Support statement: Dutch Cystic Fibrosis Foundation, the Scientific Foundation Sophia Children's Hospital and Steun door Zeevaart have given unconditional grants for the research related to this manuscript. All participating centres supplied the funding for the travel and accommodation of the site visit by the SCIFI CF team. Funding information for this article has been deposited with FundRef.

Conflict of interest: Disclosures can be found alongside the online version of this article at erj.ersjournals.com

Copyright OERS 2016 


\section{Introduction}

Computed tomography (CT) usage has increased over the past two decades to aid with diagnostic and monitoring purposes in the paediatric population [1]. CT has become an important imaging tool for patients with cystic fibrosis (CF), who develop lung disease early in life when irreversible pulmonary abnormalities can still be prevented. Some centres start monitoring the progression of early CF lung disease with CT at a young age [2-4], as this is currently the most sensitive imaging modality to detect and monitor the structural changes of early CF lung disease. Chest CT can detect structural abnormalities even before symptoms occur in a patient [2] and, in general, before it becomes apparent by spirometry [5] or any other imaging modality [6-9]. Findings on chest CT provide important timely information to CF caregivers on the presence and progression of structural lung abnormalities, and guides treatment aimed to prevent progressive pulmonary changes [10].

The sensitivity of chest CT to detect structural lung changes promptly has also resulted in several clinical trials that include chest CT as an outcome measure [11-13] and more are to be anticipated to evaluate the efficacy of new, costly, promising disease-modifying agents. Chest CT-related parameters such as bronchiectasis scores can be of use as primary or secondary outcome measures. Smaller sample sizes may suffice in studies with more sensitive CT outcome measures $[14,15]$. Chest CT can play an especially important role in clinical trials in young children, and for children and adolescents with CF, as well as in other lung diseases when sensitive outcome measures are needed [16].

To utilise chest CT in a multicentre clinical trial, it is of great importance to standardise image acquisition of chest CT scans. Acquired CT scans require sufficient image quality at the lowest possible radiation dose. Currently, technical scanning parameters and image reconstruction techniques are chosen by each centre individually, profoundly affecting image acquisition and consistency [17]. Affected image quality could indicate false disease progression or treatment effectiveness in patients. Differences can also be found not only in lung density measurements and airway analyses between scanners, but also within a specific CT scanner over time [18]. Studies have shown either overestimation or underestimation of emphysema and bronchiectasis depending on the reconstruction algorithm [17] and the inspiration level during CT acquisition [19]. Next to the variety in image quality and CT acquisition, a spread in amount of radiation dose used for each scanner and protocol can be expected. Radiation exposure comes with potential risks and it is therefore important to keep this as low as reasonably achievable, especially in young children, as they are more sensitive to ionising radiation [1]. The use of a sequential technique with gaps, i.e. a noncontiguous scan predates a volumetric or spiral scan. Currently, sequential chest CT techniques are, however, still used as a strategy to reduce radiation dose by some centres [20-22], even though this strategy comes at the cost of losing information and making longitudinal follow up less sensitive.

CT techniques have improved, with the main goal of decreasing the radiation exposure in patients while maintaining sufficient diagnostic image quality. Techniques such as automatic exposure control and the more recent development of iterative reconstruction techniques have been effective in lowering radiation exposure in paediatric patients $[23,24]$. With rapidly improving CT technology and different CT scanners, it can be a challenge to quantify and maintain consistent image quality of CT scans during a multicentre clinical trial.

The aims of our study were to characterise CT practices and the performance of CT scanners in different European CF centres involved in CF research, and to optimise CT protocols for children and adolescents. Standardised chest CT was aimed to be available for future clinical trials in the paediatric CF population. For these purposes, the Standardised Chest Imaging Framework for Interventions and Personalised Medicine in CF (SCIFI CF) was founded in 2012. Site visits were conducted at European centres, most of them being part of the European Cystic Fibrosis Society Clinical Trial Network (ECFS-CTN). We describe the current routine chest CT protocols used for children and the performance of the CT scanners using these protocols. Based on these findings, guidelines and recommendations are presented for future chest CT protocols.

\section{Material and methods}

The ECFS-CTN is a European network launched by the European CF society in 2008. It was composed of 30 selected CF sites dedicated to CF clinical research in 11 countries in 2012. All 30 ECFS-CTN centres were invited to participate with the SCIFI CF initiative. In addition to the ECFS-CTN centres, two other centres requested to participate, as they were eager to have their CT scanners characterised for research purposes. A letter was sent to each of these centres explaining the background of the SCIFI CF initiative. In addition, a questionnaire was sent to acquire general information regarding the paediatric CF centre and an inventory of the centres' CT protocols used for CF patients was requested (see the online supplementary material for full details). Centres were selected based on the commitment of the paediatric pulmonologist and radiologist, availability of the CT scanner, availability of the employees and technicians for a site visit, and consent to compensate for the costs of the site visit. To ensure the commitment of 
both the department of paediatric pulmonology and the department of radiology, a letter of agreement signed by both a paediatric pulmonologist and a radiologist was required.

All participating centres and their data are anonymised in this report.

\section{Site visit}

The visits to the selected centres were planned and executed over a period of 2 years starting from 2013. A SCIFI CF site visit was prepared in close collaboration with the CF coordinator of each centre. During the 2-day visit, a presentation about the SCIFI CF initiative was given, phantoms were scanned on the CT scanner and training was provided concerning spirometer guided CT. The feasibilities of standardised inspiration levels acquired during CT scanning have been described separately (E.R. Salomon, S. Lever, W. Kuo, et al.; unpublished observations).

\section{Evaluation of routine CT protocol}

The main CT scanner and the age-specific routine chest CT protocols used in clinical practice to acquire CT scans in children and adolescents with CF were studied and evaluated with three age-specific phantoms (QRM; Quality Assurance in Radiology and Medicine, Möhrendorf, Germany). These phantoms were designed to represent the average patient size of a newborn, a child (5 years old) and a young adult. The size and geometry of the adult phantom was based on measurements in a European, young-adult population [25]. The newborn and child phantoms are scaled down versions of this adult phantom. Based on the phantom sizes and the information available from the Image Gently campaign [26], the newborn, child and adult phantoms correspond to a patient weight of 4, 18 and $58 \mathrm{~kg}$, respectively. The materials used in these anthropomorphic phantoms mimic human tissues in the thorax with regards to the characteristics of X-ray attenuation. The tissues of the phantom include artificial lung parenchyma, bone and soft tissue-equivalent material. In addition, inserts containing homogeneous solid water, iodine or soft tissue-equivalent material can be placed on various positions of the phantom. A tungsten wire and golden disk insert were used to determine the spatial resolution of the scanner. For the child phantom, the applied X-ray tube voltage and rotation time were noted. To assess the applied radiation dose as a function of patient size, the newborn, child and adult phantoms were first scanned according to the centres' current CT protocol for patients with CF in these age groups. The scanner's radiation output was determined by the reported volumetric CT dose index $\left(\mathrm{CTDI}_{\mathrm{vol}}\right)$ in the Digital Imaging and Communications in Medicine (DICOM) header or from the accompanying DICOM dose report image. When this information was not available, the $\mathrm{CTDI}_{\mathrm{vol}}$ was determined manually, with the aid of tabulated dose values in the software package CT-Expo (version 2.0; Science and Technology for Radiology, Buchholz, Germany) using the scanning parameters of the protocol. The $\mathrm{CTDI}_{\mathrm{vol}}$ value of noncontiguous sequential CT protocols was extrapolated to the corresponding dose of a continuous volumetric CT scan.

The median dose found in the child phantom in all centres served as a reference value for the absolute dose level. It was assumed that this reference dose level provides images of sufficient quality for diagnosis as it is based on the protocols used by the centres for their clinical routine. The dose outliers are assumed to be of too good or too bad diagnostic quality. For each centre, the ratio between the newborn and the child dose, and the ratio between the adult and the child dose were determined. The median values of these ratios were multiplied by the reference value for the child phantom to obtain reference values for the newborn and young adult.

\section{CT scanner performance characterisation}

The performance of each scanner was characterised by assessing the quality of the child phantom images reconstructed with the filtered back-projection technique. The primary measure for image quality $Q_{\text {NOISE }}$ was solely related to image noise:

$$
Q_{\mathrm{NOISE}}=\frac{1}{\sigma^{2}}
$$

where $\sigma$ is the standard deviation of the image noise. Image noise was determined in each slice in a $5-\mathrm{cm}^{2}$ region of the lung parenchyma. A relatively high $Q_{\text {NOISE }}$ factor corresponds to an image with a relatively low noise level. Thus, for a given level of image contrast, a relatively high $Q_{\text {NOISE }}$ factor also corresponds to a better contrast-to-noise ratio.

It is important to realise that image quality and applied dose are interconnected. Moreover, several aspects of image quality, in particular image noise and spatial resolution, are closely linked. Therefore, the $Q_{\text {NOISE }}$ factor was extended accordingly to take both spatial resolution and applied dose into account. 
In general, a better spatial resolution corresponds to a higher noise level. The spatial resolution of an image can be described in-plane and out-of-plane by the pre-sampled modulation transfer function (MTF) and slice sensitivity profile (SSP), respectively. The measure $Q_{\text {NOISE,RES }}$ incorporates the previously derived relationship between image noise and spatial resolution [27-30]:

$$
Q_{\text {NOISE,RES }}=\frac{1}{\sigma^{2}} \cdot \frac{M^{2} \int \mathrm{d} f f^{2} \operatorname{MTF}^{2}(f)}{\text { FWHM }_{\text {SSP }}}
$$

where $M$ is the reconstruction matrix size, FWHM $\mathrm{SSP}$ is the full width at half the maximum SSP and $f$ is the spatial frequency. The integral runs from $-f_{\mathrm{N}}$ to $+f_{\mathrm{N}}$, where $f_{\mathrm{N}}$ is the Nyquist frequency of the projection data. The SSP was determined by scanning the insert containing a $10-\mu \mathrm{m}$ thick golden disk. The MTF was derived from the point spread function that was determined from the CT scan of the tungsten wire insert.

In addition to the spatial resolution, the radiation dose applied during the CT scan influences the image noise as well. The measure $Q_{\text {NOISE,RES,DOSE }}$ incorporates both the relationship between image noise and spatial resolution and the relationship between image noise and radiation dose:

$$
Q_{\text {NOISE,RES,DOSE }}=\frac{1}{\sigma^{2}} \cdot \frac{M^{2} \int \mathrm{d} f f^{2} \operatorname{MTF}^{2}(f)}{\mathrm{FWHM}_{\mathrm{SSP}}} \cdot \frac{1}{\mathrm{CTDI}_{\mathrm{voI}}}
$$

$Q_{\text {NOISE,RES,DOSE }}$ is a figure of merit, which is similar to the dose efficiency and imaging performance parameter used by another group [31]. The higher the Q-factor, the better the imaging performance of the CT scanner. A CT scanner with a relatively high $Q_{\text {NOISE,RES,DOSE }}$ produces images with a relatively low noise level at the same dose level and spatial resolution as another scanner with a relatively low $Q_{\text {NOISE,RES, }}$ DOSE factor. CT scanners with equal $Q_{\text {NOISE,RES,DOSE }}$ factors are able to produce images of equal quality with respect to noise and resolution at equal dose levels.

The $Q$-values were determined for all axial slices scanned and averaged over all slices. Based on these averaged values and corresponding scan parameters, a general recommendation was made including the radiation dose to be applied as a function of patient age and the scan and reconstruction parameters to be used. These recommendations will be discussed in the final section of this guideline.

\section{Results}

\section{Study population}

23 out of the 30 ECFS-CTN centres completed the questionnaires. 19 centres showed interest in participating in the SCIFI CF study, of which 13 paediatric centres fulfilled the requirements to participate. In addition, the CT scanner in our own department of radiology at Erasmus MC, Rotterdam, the Netherlands (also an ECFS-CTN centre) was included in this project. Aside from the 14 ECFS-CTN centres, two additional European non-ECFS-CTN centres were included. All 16 centres were visited in 2013 or 2014. The following manufacturers of the CT scanners were involved: eight were manufactured by Siemens, five by Philips, two by GE Healthcare and one by Toshiba.

\section{Evaluation of routine CT protocol}

Nine out of the 16 centres routinely monitored CF patients with a chest CT every 2 or 3 years. Seven centres only acquired chest CT scans on clinical indication. 12 centres acquired volumetric inspiratory chest CTs, of which nine included volumetric expiratory CT scans and three, noncontiguous expiratory CTs. Two centres acquired volumetric scans only on the first scan of the patient, followed by noncontiguous CT scans for follow-up assessment. Two centres never used volumetric CTs in clinical practice for the check-up of CF patients, but only scanned noncontiguously.

Applied X-ray tube voltage for the child phantom varied between centres (range $70-120 \mathrm{kV}$, median value $100 \mathrm{kV}$ ). X-ray tube rotation time varied as well (range $400-750 \mathrm{~ms}$, median value $500 \mathrm{~ms}$ ).

Radiation doses applied to the three phantoms for each centre are presented in figure 1. On average, the applied dose $\left(\mathrm{CTDI}_{\mathrm{vol}, 32 \mathrm{~cm}}\right)$ for the child phantom was $1.8 \mathrm{mGy}$. The median dose was $1.0 \mathrm{mGy}$ (horizontal line in figure 1a). The applied dose for the newborn and adult phantoms relative to the dose for the child phantom is shown in figure $1 \mathrm{~b}$. The horizontal lines in figure $1 \mathrm{~b}$ correspond to the median relative dose levels of $64 \%$ and $223 \%$ for the newborn and adult phantom, respectively. The deviation of the bars from these lines indicates how well the dose for a newborn child was reduced and, conversely, increased for an adult relative to the dose for a 5-year-old child. For instance, site 10 was in agreement with the recommended dose levels for both absolute and relative values. 

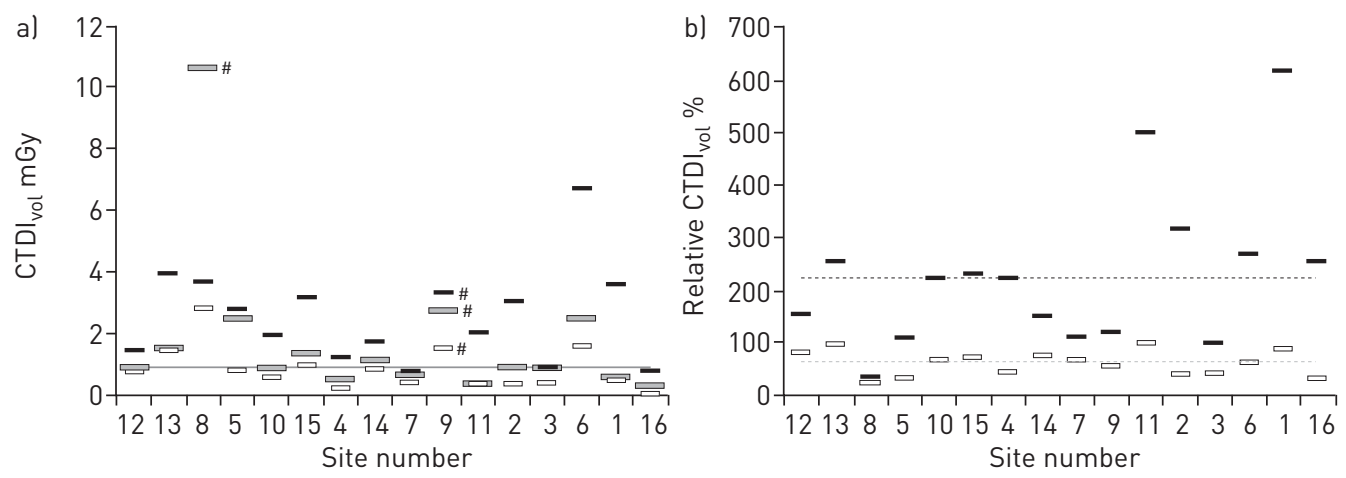

FIGURE 1 Dose levels applied in all centres. a) Volumetric computed tomography dose index $\left(\mathrm{CTDI}_{\mathrm{vol}}\right)$ applied in each centre for the child phantom (grey bars) and the median dose value (grey line), the adult (black bars) and the newborn phantom (white bars). The smaller bars represent the CTDI vol used for the newborn (white) and a

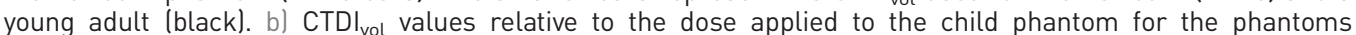
representing a newborn (white bars) and young adult (black bars). Grey and black dashed lines correspond to the recommended relative dose for a newborn and young adult, respectively. The order of the centres is the same as that in in figure 2. ${ }^{\#}$ : extrapolated CTDI vol values derived from a sequential computed tomography scan.

Young children were scanned while spontaneously breathing in 14 centres and under general anaesthesia in two centres. Starting from the age of 5-6 years old, when children can cooperate, inspiration CTs were scanned with voluntary breath hold in 15 centres and with spirometer guidance in one. Expiration CTs were scanned with voluntary breath hold in 15 centres (and in the lateral decubitus position in two) and with spirometer guidance in one.

\section{CT scanner performance}

The factors $Q_{\text {NOISE}}, Q_{\text {NOISE,RES }}$ and $Q_{\text {NOISE,RES,DOSE }}$ for each centre are shown in figure 2 relative to the average value for $Q_{\text {NOISE}}, Q_{\text {NOISE,RES }}$ and $Q_{\text {NOISE,RES,DOSE, respectively. Large variability between scanners }}$

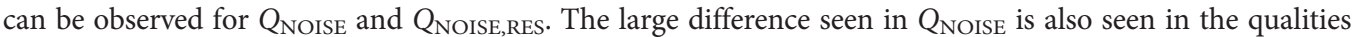
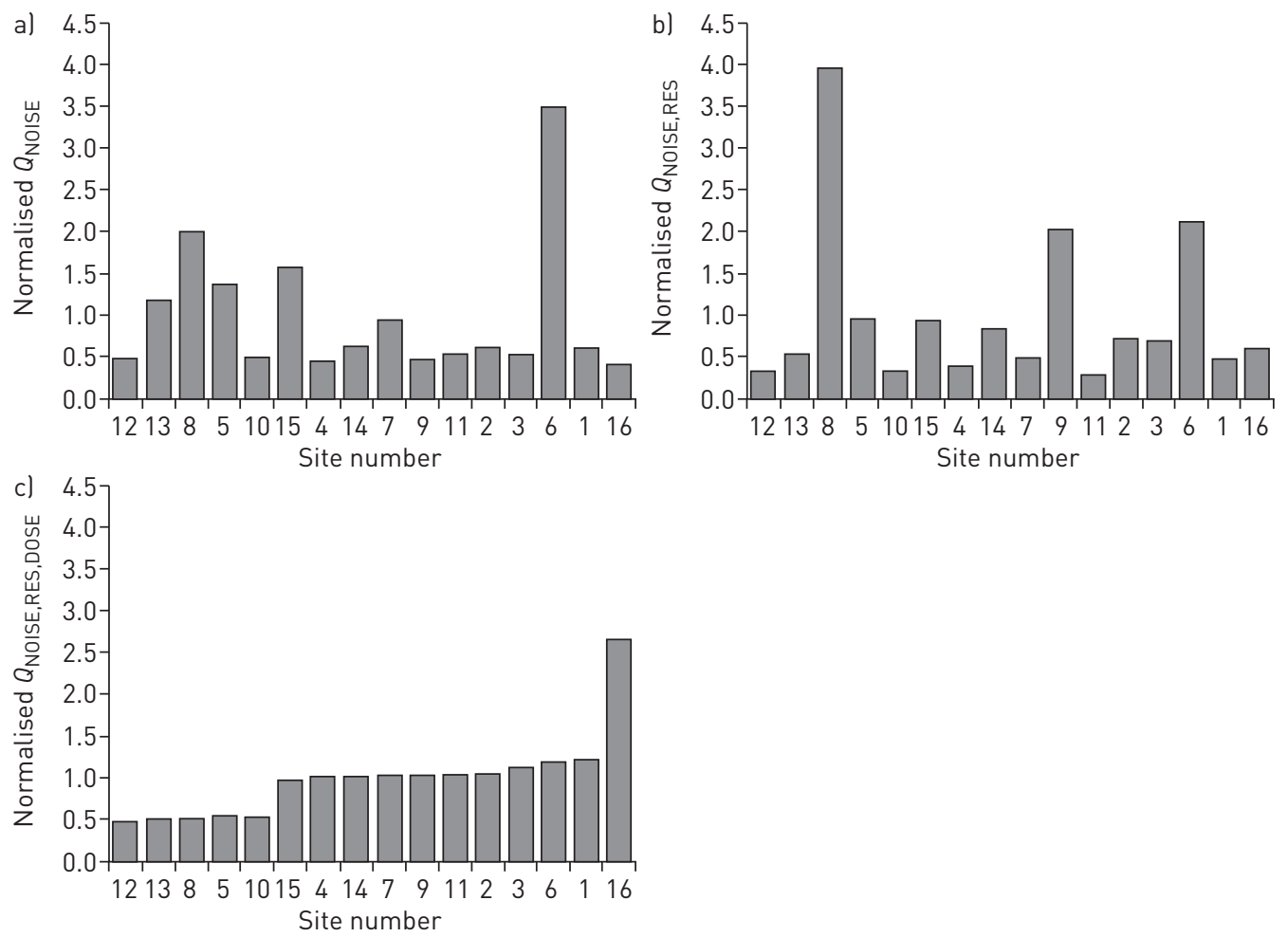

FIGURE $2 Q$ factors for each centre normalised to the average $Q$ factor. a) $Q_{\text {NOISE }}$ based on image noise. b) $Q_{\text {NOISE,RES }}$ based on image noise and compensated for differences in spatial resolution between centres. c) $Q_{\text {NOISE,RES,DOSE }}$ based on image noise and compensated for differences in spatial resolution and radiation dose between centres. Centres are sorted from small to large $Q_{\text {NOISE,RES,DOSE. }}$ 

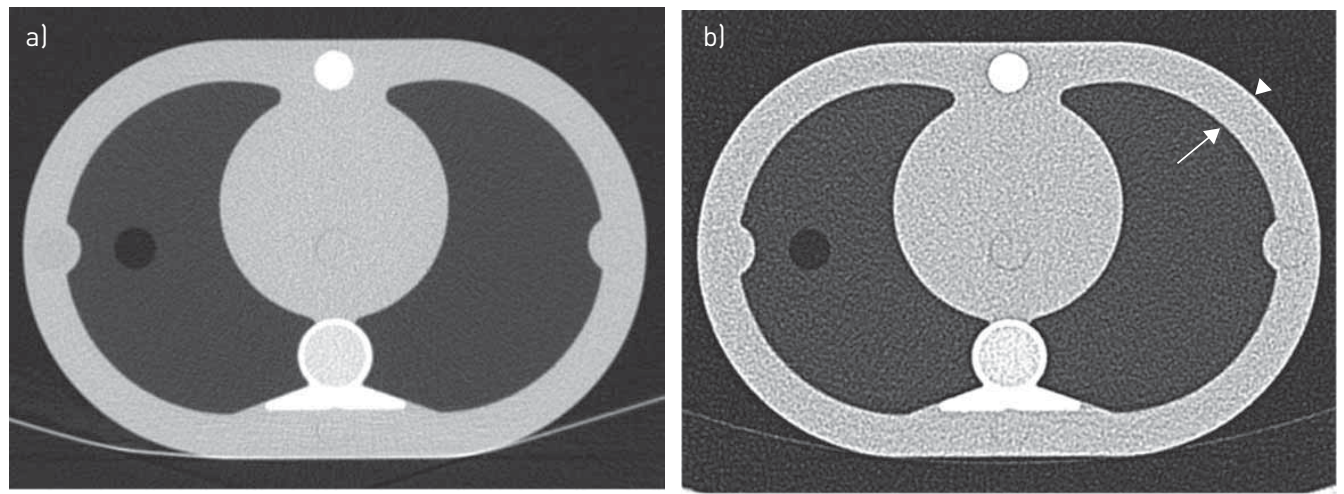

FIGURE 3 Examples of the child phantom scans made with two different CT scanners. a) The highest $Q_{\text {NoISE, }}$

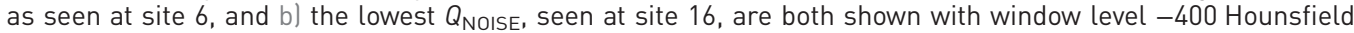
units (HU) and window width $1500 \mathrm{HU}$. Notice the influence of the edge enhancing reconstruction kernel in b): the outer edges of the phantom appear brighter (arrowhead) and outer edges of the lung equivalent tissue appear darker (arrow).

of the phantom scans (figure 3). The variability found in $Q_{\text {NOISE }}$ can partly be explained by the variability found in in-plane spatial resolution as determined by the MTF. Figure 4 shows the large variation in MTF shapes found across the centres. The influence of the MTF can for example be seen in the $Q_{\text {NOISE }}$ factor of site 9 , which is relatively low because of the relatively sharp reconstruction kernel used (site 9 in figure 2a for $Q_{\text {NOISE }}$ and the arrow in figure 4 for MTF). After correcting for the negative influence of the sharp kernel on the image noise, the $Q$-factor $\left(Q_{\text {NOISE,RES }}\right)$ increases and is even better than average (figure $2 b$ ). The latter can be explained by the relatively high dose level used by this centre (figure 1a).

After compensation for differences in dose, the figure of merit $Q_{\text {NOISE,RES,DOSE }}$ shows less variation over all centres. The performances of 10 scanners (40-128-slice scanners) were approximately equal. Thus, for a given dose level, the image quality can be standardised in the majority of the participating centres. A prerequisite is that equal reconstruction matrix size, a comparable in-plane spatial resolution and slice sensitivity profile are present.

Five scanners (16-64 slice scanners) performed below average and one scanner (>128-slice scanner) performed above average. No relation between applied X-ray tube voltage and scanner performance was found. These deviations from the average $Q$-factor might be explained by differences in in-plane sampling frequency. The scanners involved show differences in the number of detector elements per detector row and rotation time, which is directly related to the number of projection measurements per rotation. The number of detector elements per detector row is a hardware property of the scanner that cannot be changed by the user. The user can change the rotation time but options are limited. Longer rotation times do improve the sampling frequency and, thus, the performance of the scanner. This appeared to be the case for site 6 , which used a rotation time of $750 \mathrm{~ms}$ and performed better than similar scanners from the same manufacturer at rotation times of 400-500 ms. A disadvantage of long rotation times is the correspondingly longer total scan time. Alternatively, a site-specific dose level might be used at centres with scanners that perform below or above average to achieve an equal image noise level at all centres.

FIGURE 4 Variety of modulation transfer function (MTF) from all centres. The arrow indicates the MTF of site 9, with a relatively sharp reconstruction kernel. The dashed line shows an MTF when reconstructing with a kernel for quantitative image analysis. Such an MTF does not have values larger than unity.

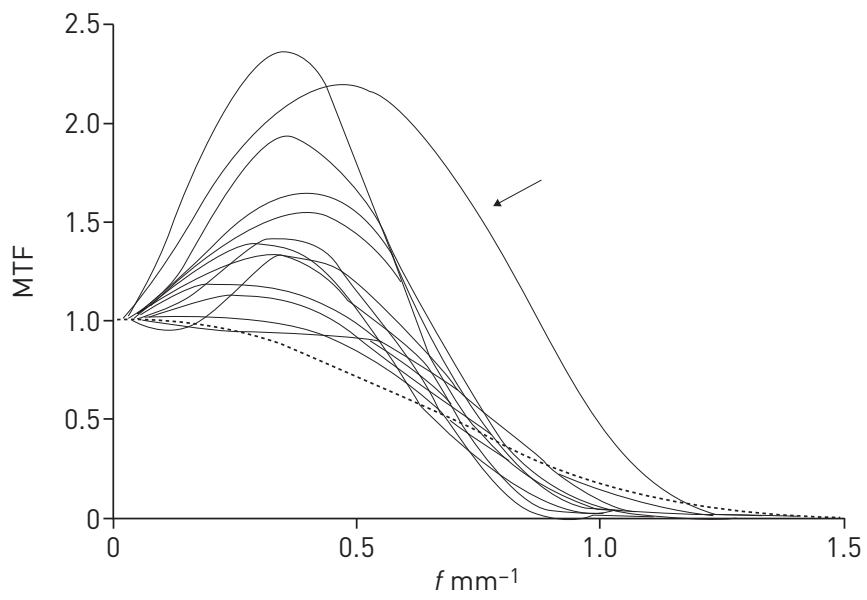




\section{Discussion}

The SCIFI CF project aimed to compare CT scanners and optimise CT protocols for children and adolescents with CF. This study showed considerable differences within CT protocols among the 16 participating CF centres. All participating centres have received general and site-specific reports including recommendations to optimise their CT protocol for (future) clinical trials. Table 1 shows the general guidelines recommended by SCIFI CF. In addition to standardising CT image quality, we explored the feasibility of standardised lung volume levels acquired during CT scanning, as observed by E.R. Salomon, S. Lever, W. Kuo, et al. (unpublished observations).

Standardisation of CT became an important aspect in clinical trials and in clinical practice. The QIBA (Quantitative Imaging Biomarkers Alliance) working group was founded to standardise CT scanners and techniques for diseases such as chronic obstructive pulmonary disease (COPD) and asthma [18]. CT standardisation has also been performed for a large observational COPD study with assessment of CT lung attenuation variation between CT scanners and the determination of longitudinal variation for individual scanners $[32,33]$. A custom designed phantom for adult patients was developed to perform quality control in CT scanners. Another study by RoBInson et al. [34], used a Plexiglas airway phantom to standardise CT protocols in children with CF. This study, however, did not have the human equivalent study material to assess the general image quality, such as image noise. Neither of these phantom studies included paediatric-sized anthropomorphic thorax phantoms to assess the automatic exposure control systems of each CT scanner.

\section{Indication and applied scan technique}

This initiative showed that $44 \%$ of the centres acquired chest CT scans only on clinical indication, while $56 \%$ of centres monitor their CF patients routinely with CT. Acquiring chest CT scans only on clinical indication could impose missed or delayed diagnosis of structural lung changes in a patient. Structural changes can be detected on chest CT scans when infants with CF do not present any clinical symptoms [16]. It is important to identify early pulmonary changes so treatment can be initiated promptly. Diagnosis of structural changes, such as bronchiectasis, was estimated to be delayed by 3 years when no routine check was performed [35].

Another finding was that four centres acquired noncontiguous chest CT scans. For CF lung disease, it has been shown that structural abnormalities will be underestimated with noncontiguous acquisition [36]. In addition, the use of limited slices will make it difficult to evaluate progression of lung disease in longitudinal follow-up or to reconstruct the image in different planes to facilitate recognition of anatomical

\begin{tabular}{|c|c|}
\hline Data acquisition mode & Volumetric, helical scan technique \\
\hline Patient position & Supine with arms above the head \\
\hline \multicolumn{2}{|l|}{ 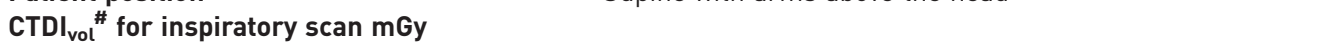 } \\
\hline 1 year old & 0.6 \\
\hline 5 years old & 1.0 \\
\hline Young adult & 2.2 \\
\hline Field of view & $\begin{array}{l}\text { As close as possible to the entirety of the lungs } \\
\text { without cutting off the lung borders }\end{array}$ \\
\hline CTDI $_{\text {vol }}$ for expiratory scan & $50 \%$ lower than inspiratory scan \\
\hline Tube voltage & $\begin{array}{l}\text { Low enough such that the recommended CTDI } \mathrm{vol}_{\text {cl }} \text { can be } \\
\text { reached (e.g. } 80 \mathrm{kV} \text { ) }\end{array}$ \\
\hline Tube current & Adapt to recommended CTDI ${ }_{\text {vol }}$ \\
\hline Pitch & $<1$, lower limit determined by maximum scan time allowed \\
\hline Slice thickness & Thinnest slice thickness (e.g. $1 \mathrm{~mm}$ ) \\
\hline Reconstruction increment & $50 \%$ overlap (e.g. $0.5 \mathrm{~mm}$ with $1-\mathrm{mm}$ slice thickness) \\
\hline Kernel for automated analysis & $\begin{array}{l}\text { Sharp reconstruction filter without under- } \\
\text { or overshoot at edges, preferably a dedicated } \\
\text { kernel for quantitative image analysis }\end{array}$ \\
\hline Iterative reconstruction technique & $\begin{array}{l}\text { If available, iterative reconstruction techniques } \\
\text { can be applied in addition to the requested filtered } \\
\text { back-projection techniques }\end{array}$ \\
\hline Shielding & Breast shielding by bismuth, for example, is discouraged \\
\hline
\end{tabular}

SCIFI CF: Standardised Chest Imaging Framework for Interventions and Personalised Medicine in Cystic Fibrosis; CTDI vol: volumetric computed tomography dose index. ${ }^{\#}$ : in $32-\mathrm{cm}$ body phantom. 
and abnormal structures of the lungs. Some centres aim to forego possible radiation risks and radiation exposure. However, with the recent improvements in scanning time and reduction in radiation exposure of volumetric CTs, the increased benefits of acquiring continuous CTs routinely justifies its use [1]. Based on the many advantages for the detection and monitoring of the heterogeneous CF lung disease we recommend acquiring volumetric CT scans in patients with CF and to forego sequential scanning [37].

Finally, chest CT was acquired during spontaneous breathing in most centres when children are non-cooperative and with voluntary breath hold when children are old enough to cooperate in most centres. General anaesthesia was only used in two centres. Since more CT scanners currently have short acquisition times, it is possible to acquire images with sufficient diagnostic quality in spontaneous breathing children. A disadvantage of this approach is that the lung will mostly be scanned at a volume near functional residual capacity, which has been recognised to reduce the sensitivity for the detection of bronchiectasis [19].

\section{Radiation dose}

Based on the dose applied to the phantoms of various sizes for all centres, recommended dose levels were derived. As the phantoms have no shoulders, these are not very representative of actual patient anatomy. In patients, tube current modulation algorithms might increase the dose in the shoulder region. The recommended dose levels here are therefore valid for the thorax region away from the shoulder region only.

The median radiation dose of $1 \mathrm{mGy}$ applied to the child phantom in all centres is in fairly good agreement with previous levels in the literature [1] and this value was typical for an ongoing multicentre trial in Australia. We advised all centres to be aware of their applied radiation doses in comparison to the median radiation dose used within the SCIFI CF initiative. For the majority of scanners, our recommendation is to consider adjustment of the radiation dose for a 5-year-old child to the median CTDI vol $_{1} 1 \mathrm{mGy}$. In this SCIFI CF initiative, we found 10 out of 16 centres differed by more than $40 \%$ from the median value of $1 \mathrm{mGy}$.

The recommended dose for the newborn child was based on the median dose for a 5-year-old child and on the median ratio between the dose for the newborn child and the dose for the 5-year-old child. Similarly, the recommended dose for the young adult was derived. Only two centres deviated by $20 \%$ or less from the recommended dose for an adult CT scan and only two centres deviated by $20 \%$ or less from the recommended dose for a newborn CT.

The Image Gently campaign provides dose reduction factors in order to establish paediatric patient diagnostic reference levels based on established levels for adult-sized patients [26]. The reduction factors are given as a function of patient size. Three series of reduction factors are provided that result in a "limited", "moderate" or "aggressive" reduction of the paediatric patient dose relative to the dose for an adult patient. Based on the "aggressive" reduction factors, the recommended dose in a newborn and adult would be $64 \%$ and $190 \%$ of the dose in a child, respectively. Thus, the relative dose recommended by Image Gently for a newborn is in agreement with our recommendations based on the median relative dose found in this study. The relative dose recommended by Image Gently for an adult patient is approximately $15 \%$ lower than our recommendation (190\% versus 223\%). The reduction factors of the Image Gently campaign were designed for a community unaccustomed to adjusting the dose for children. The "limited" and "moderate" dose reduction factors changed less with size than what we found in clinical practice in this study. Apparently, the centres involved in this study are more accustomed to adjusting the dose for children with CF than the centres that the Image Gently campaign had in mind while developing the dose reduction factors.

Doses of expiratory CTs were recommended to be 50\% lower than an inspiratory CT. A different dose level is recommended for six out of 16 scanners, to compensate for differences in performance of the scanner.

In two centres dose values were extrapolated from a noncontiguous scan. At these centres, the $\mathrm{CTDI}_{\mathrm{vol}}$ value was adjusted automatically in the scanner's dose report to reflect the dose reduction due to noncontiguous scanning. This adjusted value does not, however, reflect the dose used for a single image in case of a volumetric scan. Therefore the unadjusted $\mathrm{CTDI}_{\mathrm{vol}}$ value was used in this study instead. We do feel it is reasonable to report the relatively high unadjusted values because this makes the comparison between sites easy, as if every site uses a volumetric scan protocol and these two extrapolated dose values did not affect the median dose level of all centres. Naturally, when we reported the dose-length product as well, the sites using a noncontiguous scanning protocol would not have had such a high dose level. This report indicates, however, that these sites should consider lowering the dose per image before switching from noncontiguous to volumetric scanning. The associated loss of image quality is expected to be limited, as for the same exposure settings, image noise is different in sequential and spiral acquisition even for the same reconstruction kernel.

Some centres also discussed the use of breast shielding with a bismuth shield as a method to decrease the radiation exposure of the patient. This is not recommended as the use of a bismuth shield counteracts the automatic exposure control used in most modern CT scanners, leading to higher and unpredictable 
radiation doses [38]. Alternatively, by reducing the tube current, similar reductions in radiation dose can be achieved with equivalent image noise levels.

\section{Scanner performance}

SCIFI CF demonstrated large variations in image noise among centres (figures $2 \mathrm{a}$ and 3 ) when CT scanner protocols were not standardised. After compensation for differences in spatial resolution, differences in image noise will remain (figure $2 \mathrm{~b}$ ). When standardising, both resolution and radiation dose image quality are expected to become equal for the majority of scanners (figure 2c). Remaining differences can be compensated for by doubling the recommended dose for the five centres with a $Q_{\text {NOISE,RES,DOSE }}$ factor $50 \%$ below average. Before doing so, the actual influence of the reconstruction kernel should be assessed as the equations used in this manuscript were predictions on a theoretical model only. The same holds true when lowering the dose for the centre with a performance above average. It should be noted that image noise, spatial resolution and dose could not be fully characterised by the variables used in the equations. The standard deviation of the image noise reflects the noise magnitude only. It ignores the spatial correlations introduced into the noise by the reconstruction algorithm [39]. To minimise the effect of reconstruction filters on the Q-factor, the images reconstructed with a typical lung reconstruction filter were used for calculating the $Q$-values.

As no relationship was found between scanner performance and applied tube voltage, no recommendations on the tube voltage to be applied are given. In practice, a relatively low tube voltage may be preferred because the relatively low dose levels technically cannot be reached at high voltages.

All SCIFI CF-related results have been discussed so far in this guideline. The following is some additional recommendations concerning chest $\mathrm{CT}$ in children and adolescents based on previous literature.

\section{Selection of scan protocol for clinical studies including image analysis}

The scan protocol to be selected depends on the image analysis method that will be used. CF lung disease on chest CT has become important to compare individual patients longitudinally and cross-sectionally between patients. Several scoring techniques have been developed to assess the extent and severity of CF lung disease $[15,19,40-43]$. It has been well recognised that adequate scoring methods require a constant image quality and standardisation. Scoring techniques like the CF-CT [40] and Perth-Rotterdam Annotated Grid Morphometric Analysis for CF (PRAGMA-CF) [15] currently rely on visual assessment by an experienced observer; hence, the image quality should be on par with clinically accepted diagnostic image quality. For the CF-CT method, it is essential for CTs to be acquired volumetric in order to detect and score the complete lobes. For the SALD (severe advanced lung disease) [41] and PRAGMA-CF scoring methods, a volumetric $\mathrm{CT}$ is not required per se as long as there are at least 10 slices between the top and bottom of the lung. However, being able to visualise the entire part above and below the structure needed to be scored improves the accuracy of the different lung structures. For the airway artery method $[19,43]$, it is crucial to have thinly sliced volumetric CTs with an overlapping increment to allow measurement of the airway and artery diameters in reformatted slices perpendicular to the airway axis. Fewer slices could be an option when measuring the airway and artery dimensions in an axial, coronal or sagittal view only. Reconstructions with $0.5-1 \mathrm{~mm}$ thickness using a sharp reconstruction filter, possibly with enhancement of the edges, results in images with the impression of a higher resolution. This is needed to detect and manually delineate the smaller airways. Selection of the scoring technique is dependent on the end-point of the (multicentre) trial. Chest CTs of a multicentre trial should be scored centrally instead of at each centre separately.

\section{Automated image analysis}

Automatic image analysis methods exist and are in development for quantification of lung parenchyma [44, 45], air trapping [44] and airway properties [46-49]. These methods can replace time-consuming manual annotations and are capable of obtaining a much larger number of high-precision measurements at any point in the airway structure or lung without additional costs. Because of their automatic nature, measurements are always performed in the exact same way and will not be influenced by inter- or intraobserver variability issues.

Automatic methods rely on mathematical models that characterise the CT image properties. A high degree of homogeneity between analysed images is often required. Consequently, small variations in acquisition protocols or inspiration level, barely perceivable to the human eye, can greatly affect the obtained measurements. Therefore, standardisation of inspiration level and image acquisition protocol is very important when automated image analysis methods are used, even more so than for visual inspection. The requirements to the image acquisition protocol differ for different automated analyses. Quantification of trapped air and consolidation based on CT intensity values can be performed in thicker slice and soft reconstructions, while quantification of the airway walls, identification of the lobes and characterisation of parenchymal texture patterns benefit from thin slices and a sharper reconstruction, showing more detail. 
For all automated image analysis purposes, we recommend to store thin-slice reconstructions (slice thickness $\leqslant 1 \mathrm{~mm}$ ) with overlapping slices with a relatively sharp reconstruction kernel. The reconstruction kernel should be as sharp as possible, without under- or overshooting (over-enhancing). Then, if desired, thick-slice resampling or image denoising can be easily performed as image post-processing. Because each automatic method relies on different image assumptions, the ideal kernel may vary from technique to technique. A safe choice would be a dedicated kernel for quantitative image analysis as shown in figure 4 with the dashed line. All the lung kernels we had analysed deviated strongly from this kernel.

To conclude, in order to optimise the image properties for automated image analysis, a careful selection of reconstruction kernel and a high level of image quality standardisation are required to balance between radiation dose and image quality.

For clinical practice, there are currently no automated analysis methods available. Using a spirometer-controlled volumetric scan protocol allows comparison with great precision slice per slice to detect changes between the most recent scan and previous scans. It is strongly recommended to use structured reporting to improve consistency between and within radiologists. These reports should at least include the components used in the scoring systems [50].

\section{Iterative reconstruction techniques}

Iterative reconstruction techniques are more and more commonly applied in clinical practice. Often a dose reduction is reported when switching to these techniques [51]. Since effect of iterative reconstruction on (automated) image analysis is not yet known, it is recommended to store a second reconstruction using filtered back-projection aside from the iterative reconstruction kernel. Most importantly, large dose reductions should be avoided when compared to the recommended dose levels in table 1 .

\section{Conclusion}

In conclusion, considerable variation was found in radiation dose applied in the paediatric CF population among European centres. This difference cannot be explained by differences in scanner performance. Specific CT protocols are needed to balance radiation dose and image quality in both clinical practice and for research purposes. With increasing demand for more sensitive outcome measures in upcoming clinical trials the SCIFI CF project shows that standardisation of chest CT is required and more importantly feasible in a multicentre cohort.

\section{Acknowledgements}

The authors would like to acknowledge Elizabeth Salamon, Karla Logie, Pauline Wesselman and Els van der Wiel (Dept of Pediatric Pulmonology, Erasmus MC - Sophia Children's Hospital, Rotterdam, The Netherlands) for their involvement in setting up the SCIFI CF project and executing the site visits.

The members of the SCIFI CF study group (in alphabetic order) are Laureline Berteloot (Dept of Pediatric Radiology, Necker Children's Hospital of Paris, Paris, France), Kris de Boeck (Dept of Pediatrics, University of Leuven, Leuven, Belgium), Cesare Braggion (Cystic Fibrosis Centre, Pediatric Department, Meyer Children's Hospital, Florence, Italy), Torkel B. Brismar (Dept of Clinical Science, Intervention and Technology, Karolinska Institutet, Stockholm, Sweden), Rosaria Casciaro (Dept of Pediatric Pulmonology, Cystic Fibrosis Centre, IRCCS Giannina Gaslini, Genoa, Italy), Desmond Cox (Dept of Paediatric Respiratory Medicine, Our Lady's Children's Hospital, Crumlin, Ireland), Jane C. Davies (Dept of Respiratory Medicine, Royal Brompton and Harefield NHS Foundation Trust, London, UK), C. Kors van der Ent (Dept of Pediatric Pulmonology and Allergology, Wilhelmina Children's Hospital, University Medical Center Utrecht, Utrecht, The Netherlands), Pilar Garcia Peña (Dept of Pediatric Radiology, Hospital Universitari Vall d'Hebron, Barcelona, Spain), Silvia Gartner (Dept of Pediatric Pulmonology, Hospital Universitari Vall d'Hebron, Barcelona, Spain), Nanko de Graaf (Dept of Radiology, Erasmus MC - Sophia Children's Hospital, Rotterdam, The Netherlands), David Hansell (Dept of Radiology, Royal Brompton and Harefield National Health Service Foundation Trust, London, UK), Lena Hjelte (Dept of Clinical Science, Intervention and Technology, Karolinska Institutet, Stockholm, Sweden), Herma C. Holscher (Dept of Radiology, HAGA Teaching Hospital/Juliana Children's Hospital, the Hague, The Netherlands), Annmarie Jeanes (Dept of Radiology, Leeds General Infirmary, Leeds, UK), Pim A. de Jong (Dept of Radiology, University Medical Center Utrecht, Utrecht, The Netherlands), Ahmed Kheniche (Imaging Dept, Assistance Publique Hôpitaux de Paris, Hôpital Robert Debré, Paris, France), Timothy Lee (Leeds Regional Paediatric Cystic Fibrosis Unit, Leeds General Infirmary, Leeds, UK), Giorgio Lucigrai (Dept of Pediatric Radiology, IRCCS Giannina Gaslini, Genoa, Italy), Marco Di Maurizio (Dept of Radiology, Meyer Children's Hospital, Florence, Italy), Anne Mehl (Dept of Pediatric Pneumology and Immunology, University Children's Hospital Charité, Berlin, Germany), Anne Munck (Assistance Publique Hôpitaux de Paris, Hôpital Robert Debré, Paediatric Gastroenterology and Respiratory Dept, CF Centre, Université Paris 7, Paris, France), Marianne Nuijsink (Dept of Pediatrics, HAGA Teaching Hospital/Juliana Children's Hospital, the Hague, The Netherlands), Jacqueline Payen de la Garanderie (Dept of Radiology, Hospices Civils de Lyon, Lyon, France), David Rea (Dept of Radiology, Our Lady's Children's Hospital Crumlin, Dublin, Ireland), Isabelle Sermet (Centre de Compétences de la Mucoviscidose, Hôpital Necker-Enfants Malades, and Université Paris Descartes, Paris, France), Veronika Skalicka (Dept of Pediatrics, University Hospital Motol, Prague, Czech Republic), Florian Streitparth (Dept of Radiology, Charité University Medicine, Berlin, Germany), Philippe Reix (Centre de Ressources et de Compétences de la Mucoviscidose, UMR 5558, Hospices Civils de Lyon, Lyon, France), Marleen Smet (Dept of Pediatric Radiology, University of Leuven, Leuven, Belgium) and Hana Vitouskova (Dept of Radiology, University Hospital Motol, Prague, Czech Republic). 


\section{References}

1 Kuo W, Ciet P, Tiddens HAWM, et al. Monitoring cystic fibrosis lung disease by computed tomography: radiation risk in perspective. Am J Respir Crit Care Med 2014; 1328-1336.

2 Sly PD, Brennan S, Gangell C, et al. Lung disease at diagnosis in infants with cystic fibrosis detected by newborn screening. Am J Respir Crit Care Med 2009; 180: 146-152.

3 Stick SM, Brennan S, Murray C, et al. Bronchiectasis in infants and preschool children diagnosed with cystic fibrosis after newborn screening. J Pediatr 2009; 155: 623-628.

4 Sheikh SI, Long FR, Flucke R, et al. Changes in pulmonary function and controlled ventilation-high resolution CT of chest after antibiotic therapy in infants and young children with cystic fibrosis. Lung 2015; 193: 421-428.

5 de Jong PA, Nakano Y, Lequin MH, et al. Progressive damage on high resolution computed tomography despite stable lung function in cystic fibrosis. Eur Respir J 2004; 23: 93-97.

6 Sileo C, Corvol H, Boelle PY, et al. HRCT and MRI of the lung in children with cystic fibrosis: Comparison of different scoring systems. J Cyst Fibros 2014; 13: 198-204.

7 Puderbach M, Eichinger M, Gahr J, et al. Proton MRI appearance of cystic fibrosis: comparison to CT. Eur Radiol 2007; 17: 716-724.

8 Lee KS, Primack SL, Staples CA, et al. Chronic infiltrative lung disease: comparison of diagnostic accuracies of radiography and low- and conventional-dose thin-section CT. Radiology 1994; 191: 669-673.

9 Ciet P, Serra G, Bertolo S, et al. Assessment of CF lung disease using motion corrected PROPELLER MRI: a comparison with CT. Eur Radiol 2015; 26: 780-787.

10 Bortoluzzi C-F, Volpi S, D'Orazio C, et al. Bronchiectases at early chest computed tomography in children with cystic fibrosis are associated with increased risk of subsequent pulmonary exacerbations and chronic pseudomonas infection. J Cyst Fibros 2014; 13: 564-571.

11 Wainwright CE, Vidmar S, Armstrong DS, et al. Effect of bronchoalveolar lavage-directed therapy on Pseudomonas aeruginosa infection and structural lung injury in children with cystic fibrosis: a randomized trial. JAMA 2011; 306: 163-171.

12 Robinson TE, Goris ML, Zhu HJ, et al. Dornase alfa reduces air trapping in children with mild cystic fibrosis lung disease: A quantitative analysis. Chest 2005; 128: 2327-2335.

13 Horsley AR, Davies JC, Gray RD, et al. Changes in physiological, functional and structural markers of cystic fibrosis lung disease with treatment of a pulmonary exacerbation. Thorax 2013; 68: 532-539.

14 Tiddens HAWM, Puderbach M, Venegas JG, et al. Novel outcome measures for clinical trials in cystic fibrosis. Pediatr Pulmonol 2015; 50: 302-315.

15 Rosenow T, Oudraad MCJ, Murray CP, et al. PRAGMA-CF: a quantitative structural lung disease CT outcome in young children with cystic fibrosis. Am J Respir Crit Care Med 2015; 191: 1158-1165.

16 Stick S, Tiddens H, Aurora P, et al. Early intervention studies in infants and preschool children with cystic fibrosis: are we ready? Eur Respir J 2013; 42: 527-538.

17 Boedeker KL, McNitt-Gray MF, Rogers SR, et al. Emphysema: effect of reconstruction algorithm on CT imaging measures. Radiology 2004; 232: 295-301.

18 Coxson HO. Sources of variation in quantitative computed tomography of the lung. J Thorac Imaging 2013; 28 : 272-279.

19 Mott LS, Graniel KG, Park J, et al. Assessment of early bronchiectasis in young children with cystic fibrosis is dependent on lung volume. Chest 2013; 144: 1193-1198.

20 Bendaoud S, Remy-Jardin M, Wallaert B, et al. Sequential versus volumetric computed tomography in the follow-up of chronic bronchopulmonary diseases: comparison of diagnostic information and radiation dose in 63 adults. J Thorac Imaging 2011; 26: 190-195.

21 de Jong PA, Nakano Y, Lequin MH, et al. Dose reduction for CT in children with cystic fibrosis: is it feasible to reduce the number of images per scan? Pediatr Radiol 2006; 36: 50-53.

22 Loeve $\mathrm{M}$, Lequin $\mathrm{MH}$, de Bruijne $\mathrm{M}$, et al. Cystic fibrosis: are volumetric ultra-low-dose expiratory CT scans sufficient for monitoring related lung disease? Radiology 2009; 253: 223-229.

23 Alibek S, Brand M, Suess C, et al. Dose reduction in pediatric computed tomography with automated exposure control. Acad Radiol 2011; 18: 690-693.

24 Singh S, Kalra MK, Shenoy-Bhangle A, et al. Radiation dose reduction with hybrid iterative reconstruction for pediatric CT. Radiology 2012; 263: 537-546.

25 McCollough $\mathrm{CH}$, Ulzheimer S, Halliburton SS, et al. Coronary artery calcium: a multi-institutional, multimanufacturer international standard for quantification at cardiac CT. 2007; 243: 527-538.

26 Image gently. Image Gently Development of Pediatric CT Protocols 2014. www.imagegently.org/Portals/6/ Procedures/IG\%20CT\%20Protocols\%20111714.pdf

27 Kalender WA, Fuchs T. On the correlation of pixel noise, spatial resolution and dose in computed tomography: theoretical prediction and verification by simulation and measurement. Phys Medica 2003; 19: 1000-1012.

28 Baek J, Pelc NJ. The noise power spectrum in CT with direct fan beam reconstruction. Med Phys 2010; 37: 2074.

29 Faulkner K, Moores BM. Analysis of $\mathrm{x}$-ray computed tomography images using the noise power spectrum and autocorrelation function. Phys Med Biol 1984; 29: 1343-1352.

30 Kijewski MF, Judy PF. The noise power spectrum of CT images. Phys Med Biol 1987; 32: 565-575.

31 Lewis M, Keat N, Edyvean S. Report 06013: 32 to 64 slice CT scanner comparison report version 14. London, Centre for Evidence-Based Purchasing, 2006.

32 Newell JD, Sieren J, Hoffman EA. Development of quantitative computed tomography lung protocols. $J$ Thorac Imaging 2013; 28: 266-271.

33 Sieren JP, Newell JD, Judy PF, et al. Reference standard and statistical model for intersite and temporal comparisons of CT attenuation in a multicenter quantitative lung study. Med Phys 2012; 39: 5757.

34 Robinson TE, Long FR, Raman P, et al. An airway phantom to standardize CT acquisition in multicenter clinical trials. Acad Radiol 2009; 16: 1134-1141.

35 O'Connor OJ, Vandeleur M, McGarrigle AM, et al. Development of low-dose protocols for thin-section CT assessment of cystic fibrosis in pediatric patients. Radiology 2010; 257: 820-829.

36 De Jong PA, Nakano Y, Lequin MH, et al. Dose reduction for CT in children with cystic fibrosis: is it feasible to reduce the number of images per scan? Pediatr Radiol 2006; 36: 50-53. 
37 Loeve M, de Bruijne M, Hartmann ICJ, et al. Three-section expiratory CT: insufficient for trapped air assessment in patients with cystic fibrosis? Radiology 2012; 262: 969-976.

38 American Association of Physicists in Medicine. AAPM Position Statement on the Use of Bismuth Shielding for the Purpose of Dose Reduction in CT scanning. www.aapm.org/publicgeneral/BismuthShielding.pdf Date last updated: February 7, 2012.

39 Boedeker KL, Cooper VN, McNitt-Gray MF. Application of the noise power spectrum in modern diagnostic MDCT: part I. Measurement of noise power spectra and noise equivalent quanta. Phys Med Biol 2007; 52: 4027-4046.

40 Brody AS, Kosorok MR, Li Z, et al. Reproducibility of a scoring system for computed tomography scanning in cystic fibrosis. J Thorac Imaging 2006; 21: 14-21.

41 Loeve M, van Hal PTW, Robinson P, et al. The spectrum of structural abnormalities on CT scans from patients with CF with severe advanced lung disease. Thorax 2009; 64: 876-882.

42 De Jong PA, Nakano Y, Hop WC, et al. Changes in airway dimensions on computed tomography scans of children with cystic fibrosis. Am J Respir Crit Care Med 2005; 172: 218-224.

43 Kuo W, de Bruijne M, Nasserinejad $\mathrm{K}$, et al. Assessment of bronchiectasis in children with cystic fibrosis by comparing airway and artery dimensions to normal controls on inspiratory and expiratory spirometer guided chest computed tomography. Insights Imaging 2015; 6: Suppl. 1, S197.

44 Ciompi F, Palaioroutas A, Loeve M, et al. Lung tissue classification in severe advanced cystic fibrosis from CT scans. Fourth Int Work Pulm Image Anal 2011; 57-68.

45 Galbán CJ, Han MK, Boes JL, et al. CT-based biomarker provides unique signature for diagnosis of COPD phenotypes and disease progression. Nat Med 2012; 18: 1711-1715.

46 Lo P, Van Ginneken B, Reinhardt JM, et al. Extraction of airways from CT (EXACT'09). IEEE Trans Med Imaging 2012; 31: 2093-2107.

47 Petersen J, Nielsen M, Lo $\mathrm{P}$, et al. Optimal surface segmentation using flow lines to quantify airway abnormalities in chronic obstructive pulmonary disease. Med Image Anal 2014; 18: 531-541.

48 Wielpütz $\mathrm{MO}$, Eichinger $\mathrm{M}$, Weinheimer $\mathrm{O}$, et al. Automatic airway analysis on multidetector computed tomography in cystic fibrosis: correlation with pulmonary function testing. J Thorac Imaging 2013; 28: 104-113.

49 Fetita C, Brillet P-Y, Hartley R, et al. 3D mapping of airway wall thickening in asthma with MSCT: a level set approach. In: Aylward S, Hadjiiski LM, eds. SPIE Medical Imaging. Bellingham, International Society for Optics and Photonics, 2014; p. 90352I.

50 Larson DB, Towbin AJ, Pryor RM, et al. Improving consistency in radiology reporting through the use of department-wide standardized structured reporting. Radiology 2013; 267: 240-250.

51 Kalmar PI, Quehenberger F, Steiner J, et al. The impact of iterative reconstruction on image quality and radiation dose in thoracic and abdominal CT. Eur J Radiol 2014; 83: 1416-1420. 